\title{
REENCUENTROS EN MARRUECOS DE LOS INMIGRADOS MARROQUÍES EN ANDALUCÍA
}

\author{
Alberto Capote \\ Departamento de Geografía Humana \\ Universidad de Granada
}

\section{RESUMEN}

Las nociones de migración transnacional o circulación migratoria son muy utilizadas en los estudios sobre la inmigración internacional de los últimos años, como una característica definitoria de estos movimientos más recientes. La inmigración marroquí en España, y en concreto en Andalucía, presenta una característica específica en relación con la que reside en otros países: la distancia, la rapidez y el coste juegan a favor de los marroquíes que viven en España a la hora de realizar las visitas a Marruecos. Ahora bien, en contra de lo que cabía esperar un principio, un estudio empírico realizado en un conjunto de municipios andaluces nos revela que esta circulación no es demasiado intensa, lo que se explica, entre otros motivos, por el coste económico de las estancias e igualmente porque el espacio de vida actual transcurre en sus actuales municipios de residencia: la asistencia de los hijos al colegio, los imperativos laborales... marcan esta pauta.

Palabras claves: circulación migratoria, marroquíes, Andalucía, identidad.

\section{ABSTRACT}

The notions of transnational migration or immigration movement are widely used in recent years in studies of international migration as a defining characteristic of these most recent moves. Moroccan immigration in Spain, specifically in Andalusia, presents a specific feature in relation to other countries: the distance, the speed and the cost play for Moroccans residing in Andalusia when visiting Morocco. But against all expectations, an empirical study conducted on a set of Andalusian municipalities reveals that this movement is not too intense, which is explained, among other reasons, by the economic cost of stays and also because the current living space takes place in their current residence municipalities: the attendance of the children to school, working demands ... mark this pattern.

Keywords: immigration movement, Moroccan, Andalusian, identity. 


\section{Introducción}

La consulta bibliográfica sobre la inmigración marroquí en Francia nos revela que los inmigrados han mantenido vínculos muy estrechos con sus orígenes, independientemente de los años transcurridos desde que se inició el proyecto migratorio. Incluso al llegar a la edad de la jubilación, han continuado con sus prácticas de movilidad entre los dos países: «orgullosos de su lugar de nacimiento, también reivindican el de residencia» (Charef, 2003). Una constatación similar hace De Tapia (2002) sobre la inmigración turca en distintos países europeos (Francia o Alemania, por ejemplo): familias que después de una treintena de años en el extranjero conservan lazos de unión muy activos con la sociedad de origen. La noción de circulación migratoria que propone Simon (1995) nos remite a las relaciones que se generan entre los distintos espacios ligados a la migración -lugares de procedencia, de residencia y de tránsito- y pretende dar cuenta de la complejidad de estos movimientos en los últimos años: migraciones pendulares de trabajo, retornos por vacaciones de los inmigrantes más sedentarios, desplazamientos con motivo de eventos familiares, flujo de productos, de mercancías y de información... También se utiliza el calificativo de migración transnacional para aludir a las prácticas económicas y sociales que implican un cruce asiduo de fronteras y retienen los nexos de unión de los inmigrantes con sus áreas de origen. Ahora bien, como varios autores ponen de relieve (Escrivá y Rivas, 2005; Suárez, 2007), el transnacionalismo no constituye una característica específica de las migraciones más recientes. Basten como ejemplos los casos antes citados de la inmigración marroquí y turca en Francia, a los que podríamos añadir otros ( $\sin$ ir más lejos, la propia migración española en el país galo). Lo «novedoso» de los últimos años es la dimensión que han adquirido estos procesos transnacionales: por su carácter global (no siempre se ven implicados solamente dos países), y por la inmediatez e intensidad de los movimientos gracias al avance tecnológico, lo que ha conducido a que en la literatura especializada se hable de una «conciencia transnacional» como un nuevo modo de pertenencia o identidad del inmigrante (Castles, 2004).

La inmigración marroquí en España, y en concreto en Andalucía, presenta una característica específica en relación con la que reside en otros países: la distancia, la rapidez y el coste juegan a favor de los marroquíes que viven en España a la hora de realizar las visitas a Marruecos. En consonancia con esto, cabe pensar, en un principio, que la circulación migratoria entre los lugares de origen y de recepción se caracterice por su intensidad, al menos en lo que a la realización de las visitas a los entornos de procedencia se refiere. En este artículo presentamos los resultados de una investigación empírica llevada a cabo en distintos municipios andaluces en el marco de un Proyecto de excelencia financiado por la Junta de Andalucía («Marroquíes en Andalucía: de los espacios sociales de la inmigración a los de la movilidad»), dentro del cual el autor ha realizado su tesis doctoral. Uno de los objetivos era analizar las relaciones entre los inmigrados marroquíes en Andalucía y sus entornos familiares y territoriales en Marruecos desde tres ángulos: las visitas anuales, el envío de remesas y otras formas de comunicación. El estudio se realizó en 11 municipios de Andalucía, en cinco de los cuales se concentró la tesis doctoral del autor de este artículo: por una parte, la capital granadina, que ocupa una posición bastante peculiar en el contexto andaluz por la mayor diversidad de perfiles de los inmigrados, las características de sus proyectos migratorios, el papel relevante de los jóvenes emigrados con motivo de sus estudios, el mayor equilibrio entre sexos...; y por otra, otros cuatro municipios que ofrecen un contrapunto más próximo al modelo «general» de la inmigración marroquí en la Comunidad Autónoma Andaluza, es decir, más vinculado al ámbito laboral y muy particularmente a la actividad agrícola (Aguilar 
de la Frontera, Zafarraya, Albuñol y Lucena, municipio este último donde además se emplean en la industria del mueble).

En este artículo nos ceñimos a los resultados obtenidos en torno a la cuestión de las visitas anuales a las comunidades de origen. Nuestro interrogante principal ha sido estudiar si las relaciones con Marruecos experimentan cambios significativos con el transcurso del tiempo, es decir, conforme el proyecto migratorio se va consolidando en la sociedad receptora. Nos preguntamos si la inmersión vital del inmigrado en los distintos lugares de residencia en Andalucía se traduce en un debilitamiento de los vínculos con Marruecos o, por el contrario, éstos persisten e incluso se ven reforzados. El estudio ha combinados métodos cuantitativos y cualitativos: por una parte la realización de una encuesta en panel en tres fases repartidas en dos años (2007 y 2008) y de otra una serie de entrevistas semiestructuradas (las últimas se hicieron en 2009). Tenemos que subrayar que el objetivo que ha prevalecido en su diseño ha sido permitir una comparación socio-geográfica, a partir de una serie de indicadores para los que se precisaba de un fuerte apoyo cuantitativo, sin buscar pretensiones plenamente estadísticas de ponderación de los resultados obtenidos para cada municipio.

\section{El ritmo de las visitas a Marruecos: una circulación no demasiado intensa}

Una encuesta llevada a cabo en 2005 por el Haut Commisariat Au Plan marroquí1 revelaba que los inmigrados en España son claramente los que realizan estas visitas con más frecuencia (Douidich, 2007). Antes de pasar al examen de los resultados de nuestro estudio, es necesario tener presentes algunas precisiones metodológicas sobre la recogida de información: en primer lugar, no se estableció ningún criterio temporal en lo que a la duración de las estancias en Marruecos se refiere (éstas podían ser de varios meses o, incluso, de una única jornada); en segundo lugar, las preguntas fueron abordadas en las tres fases de encuesta, con el fin de detectar si se producían cambios significativos de un año a otro o por el contrario se reproducían las mismas pautas de movilidad (frecuencia y calendario); en tercer lugar, los periodos a los que se hizo referencia en cada fase de la encuesta fueron los años 2006, 2007 y $2008^{2}$.

Iniciamos nuestro análisis abordando los resultados obtenidos en la primera fase de encuesta (realizada entre febrero y mayo de 2007). En ese momento, la gran mayoría de los encuestados afirmó haber realizado al menos una visita a Marruecos durante el año 2006 (82,6\%, 176 de 213). De éstos, la mitad (50,6\%) lo hicieron una única vez, el 30,7\% en dos ocasiones y el 18,8\% como mínimo tres veces. Expresado en otros términos, el $41 \%$ pasó al menos dos estancias en Marruecos en el periodo indicado. En lo que respecta a los datos desagregados por sexo, el porcentaje de respuestas negativas es mayor en los hombres $(22 \%)$ que en las mujeres $(8 \%)$. Este margen de diferencia se justifica, fundamentalmente, porque la mayor parte de quienes se encontraban entonces en situación irregular eran varones. Asimismo tenemos que hacer referencia a la actividad ocupacional. Las mujeres al cuidado del hogar tienen, por lo general, más disponibilidad que los maridos para viajar a Marruecos en los periodos de vacaciones escolares. Aunque no fueron casos muy frecuentes, durante el trabajo de campo comprobamos que en algunas familias sólo viajaron las madres acompañadas de los hijos. Igualmente conocimos otras situaciones

1 «Enquête de 2005 sur l'Insertion Socio-économique dans les pays d'accueil ». La encuesta se aplicó a un total de 3000 hogares y se desarrolló durante los meses de agosto y septiembre de 2005.

2 Desde enero de dicho año hasta el momento en el que se aplicó el último cuestionario, que pudo ser octubre, noviembre o diciembre de ese año. Con fines operativos, sólo hemos tomado en cuenta los desplazamientos realizados hasta septiembre de 2008 . 
en las que, si bien se desplazó la familia completa, la visita del padre duró menos tiempo por imperativos laborales. A este respecto es interesante resaltar que algunos encuestados declararon no poder agrupar los días de vacaciones disponibles y así alargar la estancia en Marruecos. Es el caso, por ejemplo, de los trabajadores de la industria del mueble en Lucena. En efecto, no siempre la prioridad es efectuar varias visitas al año a los municipios de origen, sino concentrarlas en una más larga (por ejemplo, en torno a un mes de duración en periodo estival). Durante el trabajo de campo incluso encontramos algún que otro caso de personas que a la vuelta de vacaciones se enfrentaron a la pérdida del empleo por haber excedido, supuestamente, el número de días de permiso concedido. Dicho de otro modo, las condiciones de determinados puestos de trabajo pueden suponer un límite a la hora de organizar los desplazamientos anuales a Marruecos. Podemos citar como ejemplo el caso de este joven de 33 años originario de Beni Mellal: «Bueno, me fui a Marruecos de vacaciones y llevaba todo el tiempo trabajando, todo el año. Me fui a Marruecos, pero no tengo mucho tiempo para estar allí. Llevo todo el año trabajando, por ejemplo, pero sólo me dan 15 días de vacaciones, nada más. 15 días es muy poco para estar con la familia, por eso me fui un mes y cuando volví, tengo una carta de la seguridad social en donde se me da de baja. Pregunto, ¿por qué me ha dado de baja?, y me dice que no puede esperar un mes entero a que venga, que no puede cerrar mi puesto...». El joven aseguró haber acordado con el empleador antes de la partida el número exacto de días de vacaciones.

La mayor disponibilidad para prolongar la estancia en los municipios de origen de las mujeres marroquíes de un determinado perfil (casadas, amas de casa y sin profesión) en comparación con los hombres, se puso también de manifiesto en la recta final de la investigación con algunas familias en las que se decidió que la esposa y los hijos permanecieran en Marruecos de manera indefinida como una respuesta provisional a las dificultades sobrevenidas por la pérdida de empleo del padre.

Las respuestas variaron también dependiendo de la edad de los encuestados: cuanto más jóvenes mayor es la proporción de los que no viajaron a Marruecos en 2006, aunque las diferencias no sean muy pronunciadas. De nuevo aquí tenemos que hacer referencia a la situación administrativa, puesto que las personas que en los primeros meses de 2007 se encontraban sin permiso de residencia -poco numerosas-eran esencialmente menores de 35 años. El grupo de edad en el que se registra una mayor movilidad (al menos dos estancias en Marruecos en 2006) es el que corresponde al intervalo de los 25 a los 34 años. En ellos cabe distinguir una casuística de los movimientos algo más variada que en el resto: por vacaciones, para alguna gestión administrativa relacionada con un reciente matrimonio, por trámites asociados a una reagrupación familiar, por enfermedad de un próximo... Estos últimos viajes, motivados por alguna gestión personal, suelen ser más cortos e improvisados. Aquí se incluyen personas que se hallaban en fase de reagrupar a la familia en España. Baste como testimonio la respuesta que dio este joven (33 años en 2009, casado y con esposa e hijo residiendo en Marruecos) cuando se le preguntó cuántas veces al año se desplazaba, por lo general, a su municipio de origen situado en la provincia de Beni Mellal: «Normalmente una, pero últimamente empiezas, te quitas de un problema, te empieza otro. Tengo que viajar. Nada más que me llaman, me voy. Porque mi padre es viejo. Cuando es algo de trabajo, alguien que está enfermo. Este año me fui a la operación de mi padre por el ojo, que ha perdido la vista. He vuelto aquí, se pone la mujer enferma. He vuelto otra vez. Que está embarazada y enferma y eso [la esposa], que estamos pensando [incluso] en su muerte también, ¿sabes?, y gracias a Dios que todo al final salió feliz». Este extracto de entrevista nos remite a otro aspecto de bastante interés: la valoración que los inmigrados hacen de la proximidad geográfica con Marruecos no reside tanto en que prevean realizar varios viajes al año, sino en la posibilidad de llevarlos a cabo de manera espontánea, de 
un día para otro, si se dan situaciones similares a las que hace alusión el entrevistado. Se podría decir que la cercanía les proporciona un mayor sosiego ya que les hace más llevadera la distancia: en cualquier momento, de manera prácticamente inmediata, en el mismo día (si el trabajo lo permite), pueden reunirse con los familiares en Marruecos sin necesidad de un gasto de transporte excesivamente elevado.

La situación familiar en los municipios de residencia en Andalucía guarda en gran medida una estrecha relación con la fase migratoria en la que el inmigrado se encuentra. La mayoría de los encuestados, particularmente los varones, siguieron un esquema bastante similar en el que emigraban solteros, al cabo de un tiempo contraían matrimonio y, después, una vez reunían los requisitos necesarios, procedían a la reagrupación familiar. Igualmente reparamos en la existencia de otras situaciones que escapaban a este patrón general: hacemos referencia aquí a hombres que habían optado por mantener a la esposa y los hijos (o parte de ellos) en los municipios de origen o mujeres que emigraron después de enviudar o de un divorcio y vivían también separadas de sus vástagos. Estos casos entrarían en el prototipo de lo que la literatura especializada denomina «familia transnacional», aquí en su dimensión estrictamente nuclear. Según los resultados de nuestra encuesta, las prácticas de movilidad asociadas a los retornos temporales difieren notablemente en función de la situación familiar. En términos relativos, son las personas casadas que no han reagrupado a su familia nuclear (total o parcialmente) los que más asiduamente viajaron a Marruecos en 2006. Aunque no es un perfil muy extendido entre los encuestados, cabe distinguir dos situaciones dentro de este grupo: por una parte, jóvenes con pocos años de matrimonio y en proceso de gestionar la reagrupación familiar; y por otra, los casos a los que se ha hecho alusión al inicio de este párrafo, es decir, familias nucleares más o menos maduras con miembros en uno y otro país (generalmente por propia decisión).

En lo que concierne a los solteros, quizás, de partida, cabía haber esperado más desplazamientos por la libertad que otorga para la movilidad el hecho de no tener responsabilidades familiares directas. No obstante, es de destacar que el $40 \%$ pasó al menos dos temporadas en Marruecos en 2006. También entre estos jóvenes nos encontramos con distintas tesituras que significan un freno a la hora de viajar a las áreas de procedencia: en unos casos por encontrarse en situación irregular; en otros, particularmente en la capital granadina, ya vimos cómo algunos de los estudiantes encuestados aprovechaban los periodos de vacaciones para emplearse en alguna actividad temporal; otros, en lugar de viajar a Marruecos, optan por explorar el territorio, visitar a otros familiares o amigos que residen en distintos puntos de la geografía española e incluso en otros países europeos. En otras palabras, estos últimos casos corresponden a jóvenes que no han cesado en su búsqueda de nuevos objetivos, alternativas en otros lugares o simplemente expresan interés por conocer algo distinto.

\section{Un «invitado» y un «emigrado»: percepciones de los inmigrados en los lugares de origen durante los reencuentros de temporada}

Las entrevistas semi-estructuradas nos proporcionan matices interpretativos de interés y permiten algunas reflexiones de carácter general sobre el modo en que el inmigrado vive cada año el rencuentro con el que era su espacio de vida habitual antes de emigrar. El arraigo en los municipios de instalación en España, después de varios años de permanencia, puede llegar a ser tan sólido que durante las estancias temporales en Marruecos se manifieste en el emigrado un cierto desasosiego por volver al que constituye ahora su lugar de residencia. Estamos hablando aquí de personas con una cierta estabilidad en lo que respeta a su situación administrativa, laboral y familiar, y que cuentan además en España con una red 
social relativamente diversificada. El ámbito relacional pre-emigratorio es percibido, durante esas temporadas en Marruecos, de manera distinta, no sólo por las experiencias acumuladas en el extranjero, sino también por la imagen que los familiares y otras personas cercanas proyectan sobre el propio emigrado: «Porque, no sé, uno ahora está acostumbrado aquí [...]. Nos vamos de vacaciones, llevas 20 o 25 días y ya tienes ganas de volver aquí. ¿Qué vas a hacer?, me pasa eso mucho a mí. El año pasado estuvimos allí un mes y he llegado, ya quería venirme. No te lo puedes imaginar. Sí, tengo ganas de volver aquí [...]. No, es por otra cosa, de verdad, es que aunque allí yo he vivido muchos más años que aquí, pero eso ahora es de paso. Ahora estamos aquí, uno está acostumbrado a trabajar y todo eso y si uno se queda, por ejemplo, un mes o dos meses sin trabajar, se aburre. No puede hacer nada ni..., aunque te vas de viaje, pero no me voy a ir para pagar, para sacar de los bolsillos, sin meter nada». "Esto te tira. Tú en tu mismo país no vas a descansar. Por ejemplo, si llevas aquí 20 años, él tiene su lugar aquí donde se descansa. Tiene su casa donde tiene todo preparado... Un invitado, en Marruecos un invitado. Un mes, llegas y no puedo aguantar y me voy. Me voy, que no puedo aguantar».

La actividad laboral, como se advierte en el primer testimonio, constituye, bastante a menudo, una de las principales objeciones a la hora de desplazarse o prolongar las estancias en Marruecos. En determinados casos esto se podría considerar hasta cierto punto un signo de instalación consistente en los municipios de residencia en Andalucía. Incluimos aquí, por ejemplo, quienes tienen una actividad agrícola en régimen de arrendatarios o de medianeros, en los municipios de Albuñol y Zafarraya. Las labores de mantenimiento de la tierra junto con las fases de recolección mantienen a estas personas ligadas al trabajo durante la mayor parte del tiempo. Las estancias en las comunidades de origen representan, eminentemente, el momento del año en el que se produce el rencuentro con los familiares, en particular con los padres. La poca inclinación que expresan estas personas a prolongar las temporadas en Marruecos no debe interpretarse como una desvinculación voluntaria de la sociedad de origen, sino más bien como un signo de su radicación en sus territorios de residencia en Andalucía: allí donde trabajan, han adquirido posiblemente una vivienda, los hijos van al colegio, han generado una nueva red social que se extiende a su vez a la de los propios descendientes adolescentes... Dicho de otro modo, «la consolidación de las condiciones socioeconómicas de integración del inmigrado en su medio de instalación puede obstaculizar las visitas al país de origen» (Cohen y Capote, 2011: 261). También es oportuno subrayar que algunos entrevistados afirmaron que sus hijos mostraban mayores reticencias a dilatar las estancias en Marruecos a medida que crecían, por el simple hecho de que preferían compartir el tiempo de vacaciones con sus amistades en España: «Cuando bajo a mí me gusta, me gusta mucho Marruecos. Pero, lo que pasa, que bajo y estoy un mes y ya en cuanto pasen dos o tres días del mes, ya me agobio, quiero venirme para acá. Bajo a ver cuando hay mucha gente y amigos que conozco. Pero luego, cuando paso más de un mes, no puedo estar más allí [...].No sé, es diferente. Bueno... o cuando se van los amigos y me quedo sola, no puedo estar allí...» (hija de 18 años de uno de los entrevistados).

La apreciación que hizo el padre de esta última interlocutora de sentirse un emigrado, un invitado, en definitiva una persona distinta de la que partió, la expresaron también algunos jóvenes, principalmente urbanos. Se repite con bastante frecuencia en los relatos el hecho de que las antiguas amistades también hayan emigrado, ya sea en el interior de Marruecos o a un país distinto. Estas ausencias son un factor que contribuye a que el contexto relacional anterior a la partida se perciba ahora extraño: «No lo sé. Estaba andando en las calles así, ves la gente y digo yo no pertenezco, como si no pertenezco a esto, no soy de aquí. No sé, no sé por qué. Pero, tal vez sería debido a que la mayoría de mis amigos 
no estaban. Pues, cada uno donde estaba, bien en Alemania, en Bélgica, en España, en Francia, cada uno donde estaba. No sé, pero me sentí como si... Luego lo que quería era ver la familia nada más».

Schaeffer (2002), en su estudio sobre los retornos de los inmigrantes marroquíes en Francia durante los meses de verano, llega a la conclusión de que éstos experimentan durante estas estancias en Marruecos un proceso dual en la percepción que tienen de su posición social en los espacios de vida anteriores a la emigración: por una parte, no se desvinculan, por lo común, de los valores y las tradiciones de la sociedad en la que nacieron y transcurrió una parte esencial de sus vidas; por otra, al mismo tiempo, la consideración de turistas (o de emigrados) con la que se perciben les permite igualmente marcar una cierta diferenciación social. De los relatos de nuestro estudio se desprende que los lazos familiares representan uno de los principales acicates para preservar los vínculos con la sociedad de origen, lo que se traduce en el mantenimiento de ciertos valores y costumbres, por ejemplo en relación con el matrimonio o las celebraciones de carácter religioso (a menudo también fruto de una presión social subyacente en los lugares de destino por parte de otros connacionales). En lo que respecta a la «distinción social», ésta se afirma tanto en la propia proyección que el inmigrado quiere reflejar de su experiencia migratoria durante las vacaciones como por las expectativas forjadas entre los familiares cercanos. «Es que cuando nos vamos alli [a Marruecos], toda la familia se reúne, tiene que gastar él todo, hacer la compra y todas esas cosas, muchos gastos y el dinero se va rápido», relataba la esposa de uno de los hombres entrevistados, oriundo de la provincia de Nador, a lo que añadía: «Sí, a mí no me importa lo que digan allí, aunque a mi marido si le importa mucho. Sí, a él le preocupa mucho la imagen, a mí no, yo al contrario. Él me compra ropa cuando voy allí para que me vean bien». Son días de reunión con la familia y también de mostrar ante la comunidad en su conjunto los logros del proyecto migratorio. Esta pretensión de querer desmarcarse de lo que se considera la situación dominante en el grupo - hacer visibles los éxitos alcanzados en el extranjero - la han expresado otras personas recurriendo a los recuerdos de la infancia y la adolescencia, cuando eran ellas mismas los testigos de la llegada de los emigrantes durante los meses estivales: «Pues, nada, gente que trabaja en Europa y que venían, les decían los inmigrantes. Allí en Marruecos también les decían inmigrantes, «vacacioneros», que vienen de vacaciones. Gente que vive aquí en Europa y viene de vacaciones. Tienen más dinero, eso sí lo sabe todo el mundo. Porque vienen siempre con coche de lujo, vienen siempre bien vestidos. Eso sí. Pero la gente, ¿qué va a pensar?, esta gente tiene dinero. Esto sí. Pues, cultos, no te puedo decir que eran muy cultos. Pues, yo los veía menos cultos que yo, ¿qué quieres que te diga?, yo tenía tíos y todo, pero... menos cultos, menos cultura, menos civilización, menos que... Hay gente que se notaba que estaba cerrada y que venía con ropa nueva, con coche y todo. Pero tú te das cuenta que es una persona que está cerrada. Por ejemplo, yo tenía unos cuñados que eran de allí, casados con mis tías, que llevaba casi 20 años en Francia y que no sabían hablar francés».

Otro elemento que los inmigrados tienen que considerar al organizar sus visitas a Marruecos es el coste económico que éstas conllevan, sobre todo en aquellas familias que hacen una única estancia anual, la cual representa uno de los momentos más destacados del año. El presupuesto puede llegar a ser bastante elevado: a los gastos asociados al transporte hay que añadir los de la estancia, los regalos y la provisión económica que normalmente se suele aportar. Retomando el testimonio anterior de la esposa de uno de los entrevistados, cuando se le preguntó con cuánto tiempo de antelación iniciaban los preparativos del viaje anual, la respuesta fue inmediata: «Un año. Para ahorrar, para comprar regalos, para comprar poco a poco, para llevarle a cada persona a quien quieres o a cada persona 
que conoces que puede venir a verte...» ${ }^{3}$. De este modo aprovechan las temporadas de rebajas, las ofertas que van encontrando..., lo que se podría considerar otra señal de cómo la familia en Marruecos está presente durante todo el año en la vida de los inmigrados, a pesar de la distancia física.

Llegados a este punto, hay que examinar también la situación ocupacional y su influencia en la gestión y la periodicidad de las visitas a Marruecos. No sólo por el coste económico que implica realizarlas, como acabamos de decir, sino también porque la disponibilidad temporal puede diferir sustancialmente según los trabajos. En una situación de inestabilidad laboral, caracterizada por la sucesión de empleos temporales, las estancias en Marruecos son más difíciles de organizar. Los periodos de actividad se intercalan con los de búsqueda, dificultando así las previsiones. Es el caso, por ejemplo, de uno de los hombres entrevistados en Granada. Oriundo de la provincia de Nador, trabajó como cocinero en distintos restaurantes durante varios años: "Porque te digo... estaba trabajando en restaurantes y siempre cuando hay tiempo, no hay dinero. Y cuando hay dinero, no hay tiempo y también con los niños en invierno están en el colegio y en verano ellos están en vacaciones, yo estoy trabajando. Tengo que trabajar en verano, la temporada de los restaurantes. Eso también, nada más. Y otro motivo porque venían mis padres también. Me convenzo diciendo que ya que no puedo ir a ver a mis padres, ellos vienen a verme a mí».

Los resultados en nuestra encuesta indican que los trabajadores autónomos son los que realizaron un mayor número de desplazamientos entre Marruecos y Andalucía en el año 2006. Concretamente, se trata de comerciantes que, además de poseer un establecimiento propio, se dedican a actividades de importación de productos y su posterior distribución entre otros miembros de la profesión. Estos casos nos hacen recordar las prácticas de movilidad estudiadas por Tarrius (2002) relacionadas con las actividades comerciales de la población inmigrada en distintas ciudades del Mediterráneo. Sin embargo, no es sencillo establecer un paralelismo. No se aprecia entre nuestros interlocutores en Granada la identidad nómada de la que habla el autor para referirse al hábito de estos comerciantes de moverse entre distintos lugares, generándose entre ellos una especie de «conciencia o identidad transnacional». Los desplazamientos detectados en la capital granadina se repiten a lo largo del año, en determinados casos con mucha asiduidad. Pero este trasiego no impide que estas personan consideren que su actual hogar de residencia está en Andalucía: con bastante frecuencia son hombres que han reagrupado a sus familias, han adquirido una vivienda en propiedad y presentan otros signos de estabilidad.

Los resultados de la encuesta muestran también que los trabajadores agrícolas son los que con menor frecuencia efectuaron desplazamientos a Marruecos en 2006: sólo uno de cada cinco lo hizo en más de una ocasión. No podemos olvidar que determinadas campañas agrícolas coinciden con períodos clave del calendario festivo: es el caso de la recogida de aceitunas en Lucena y Aguilar de la Frontera durante las vacaciones de Navidad. Algunos años incluso puede llegar a cubrir la Semana Santa. Asimismo, hay que tener presente que el empleo en este sector se caracteriza por su discontinuidad, lo que nos remite a las migraciones estacionales dentro de España en busca de ingresos complementarios. El hecho de ir enlazando unas campañas con otras, intercalando periodos de jornadas de trabajo dispersas, se traduce en que las visitas a Marruecos se condensen muy a menudo en una sola estancia. Los viajes tienen lugar en aquellos periodos en los que son muy escasas las posibilidades de conseguir peonadas adicionales. Igualmente cabe preguntarse si los recursos de un jornalero agrícola dan para cubrir varios viajes al año, máxime cuando, además, muchos

3 Una constatación similar hace Charef (1999) entre los inmigrados más jóvenes de origen susí: el precio del viaje, los regalos que se ven comprometidos a aportar, los gastos que efectúan durante su estancia, entre otras razones, les inducen a disminuir la frecuencia de las visitas. 
de ellos envían remesas a sus familiares. La duración de las visitas, los días exactos de la partida y el regreso... dependen, como nos describe este joven entrevistado en Riofrío, provincia de Granada (previamente encuestado en Zafarraya), de cómo se hayan desarrollado las grandes campañas agrícolas: «Si sale la cosa bien, acaba la temporada bien en Zafarraya, la mayoría, el 90\% van a Marruecos, todos. Si sale un año arruinado como el año pasado, el año anterior, se va la gente a recuperarse a otro sitio [...]. O se van de Zafarraya directamente una vez que se acaba la temporada de Zafarraya, si ha escapado bien o medio regular de eso, como decimos que se van para pasar un poco de tiempo alli».

Como se preveía desde un principio, la distancia geográfica en relación con las áreas de procedencia es otro factor que influye en la intensidad circulatoria entre los dos países. Tanto si adoptamos el criterio del lugar de nacimiento como el de última residencia antes de emigrar al extranjero, son los oriundos de las provincias del Marruecos central los que con menos frecuencia se desplazaron en el curso del año 2006. Además, arrojan el porcentaje más alto de respuestas negativas, posición que se justifica por la presencia de jóvenes en situación irregular originarios de provincias como Beni Mellal o El Kelaa. En el lado opuesto encontramos a los que proceden de la Península Tingitana y del conjunto Rif-Oriental. Por ejemplo, mientras que el 56,5\% de los nacidos en la región de Yebala viajó al menos dos veces en 2006, este porcentaje en el Marruecos central es del 26\%. Evidentemente, no es lo mismo desplazarse a Beni Mellal (a $630 \mathrm{~km}$ de distancia de Tánger por carretera) que a Casablanca (a $341 \mathrm{~km}$ ) o Larache (a sólo $86 \mathrm{~km}$ ). Pero tampoco podemos pasar por alto que los oriundos de las provincias interiores, como las citadas anteriormente, se emplean mayoritariamente en el sector agrícola (por ejemplo en Zafarraya), que suelen pasar una única temporada al año en Marruecos, salvo situaciones excepcionales (enfermedad de un familiar, cuestiones administrativas por resolver...). En este último caso se desplaza una sola persona, y no la familia al completo. Por el contrario, como ya hemos visto, los perfiles de las personas procedentes de las otras regiones migratorias son más heterogéneos: encontramos una mayor presencia femenina (incluyendo a mujeres que emigraron con un proyecto que podríamos considerar «autónomo», lo que no ocurre, por ejemplo, entre las encuestadas oriundas de la provincia de Beni Mellal), mayor diversificación también en lo que a los sectores de ocupación se refiere, sin olvidar a los jóvenes estudiantes, particularmente aquéllos que proceden de las provincias del norte (Alhucemas, Nador...).

\section{La circulación migratoria al inicio de la crisis económica}

Otro de nuestros objetivos de partida era conocer si la organización que hacen los inmigrantes de sus visitas a Marruecos sigue un patrón que se repite cada año en lo que respecta a su frecuencia y sus fechas. En la figura 1 hemos representado los porcentajes de respuestas positivas que dieron los inmigrados que atendieron a las tres oleadas de la encuesta. Recuérdese que se hacía referencia a tres años consecutivos: 2006 (que ya hemos visto), 2007 y 2008. En dicha figura aparecen dos curvas: la que hemos denominado «A» tiene en cuenta los valores obtenidos para el total de personas que participaron en cada oleada (por tanto, el tamaño muestral en los tres momentos es distinto), y la «B» los datos correspondientes a aquéllas que lo hicieron, sin excepción, en los tres pasos. Como podemos comprobar, las dos líneas siguen una evolución muy similar con porcentajes que no difieren sustancialmente. Entre los años 2006 y 2007 se advierte muy poca variación en los resultados, aunque se detecta una ligera subida en las respuestas positivas. Aquí se incluyen algunos casos de personas que entre la primera y la segunda fase lograron regularizar su situación administrativa y pudieron llevar a cabo su primera visita a Marruecos desde su llegada a España. Sin embargo, el porcentaje disminuye notablemente en 2008. No podemos ignorar 
que este último año no se cubrió completamente, puesto que el cuestionario fue aplicado en el último trimestre. Así pues, no se tuvieron en cuenta los desplazamientos que pudieran realizarse con motivo de la fiesta del Aid El Kebir, que en 2008 correspondió a primeros de diciembre. Debemos tomar la misma precaución con respecto a la frecuencia con la que efectuaron los desplazamientos: se han ido incrementando los casos de personas que sólo viajaron una vez, situándose el cambio más brusco entre 2007 y 2008 (figura 2). Pero, por otra parte, como veremos más adelante, en los tres años analizados el grueso de las visitas a Marruecos tuvo lugar durante los meses de verano. Raramente, cuando se hace un único viaje, la fecha elegida es el último trimestre del año. En este sentido, el calendario de la tercera fase de encuesta no ha debido «deformar» en exceso el perfil obtenido.

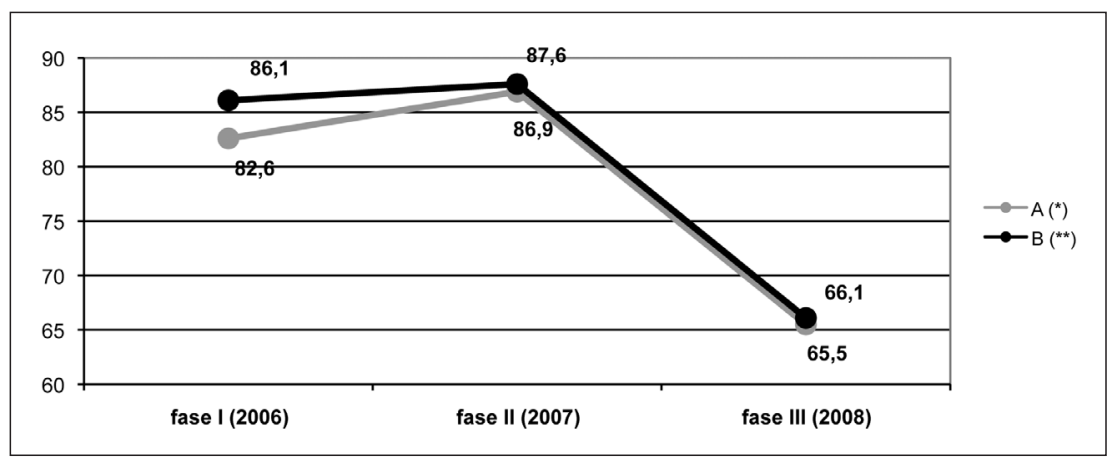

FIGURA 1. Porcentajes de respuestas positivas a la pregunta de si se ha viajado o no a Marruecos en 2006, 2007 y $2008(\%)$.

Fuente: Encuesta DINAMO (Dinámicas de la movilidad espacial).

* Total de respuestas en cada fase de encuesta

** Respuestas de las personas que han participado en las tres fases de encuesta

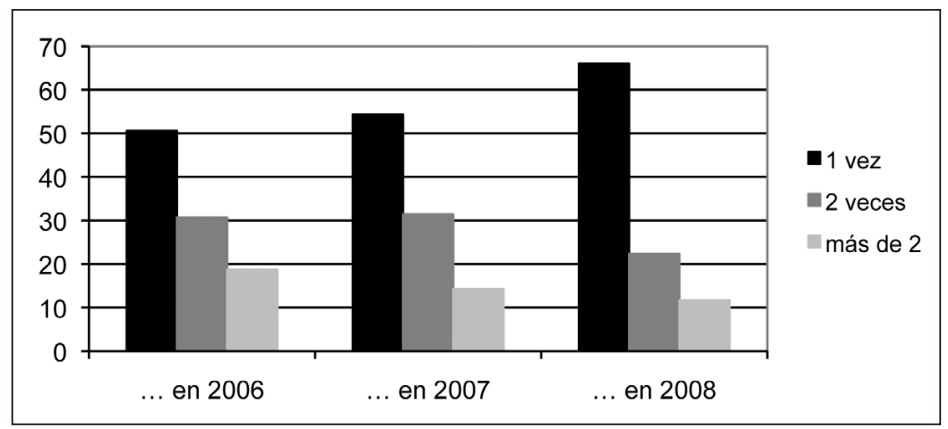

FIGURA 2. Frecuencia de las visitas realizadas a Marruecos 2006, 2007 y 2008 (\%)

Fuente: Encuesta DINAMO.

* Total de respuestas en cada fase de encuesta

Estas apreciaciones llevan a preguntarse hasta qué punto el cambio en el clima económico a partir del año 2008 ha repercutido en los resultados de la recta final del trabajo de campo. Dicho con mayor precisión, la disminución de las respuestas positivas, ¿está dando a entender que en un contexto de empeoramiento económico los inmigrados afectados 
interrumpen las visitas a la familia para reducir gastos? Las respuestas a esta pregunta no van, de nuevo, en una única dirección.

En los dos últimos cuestionarios, en caso de contestación negativa, se preguntaba por los motivos de no haber ido a Marruecos en el año de referencia. En 2007 la respuesta más común señalaba razones administrativas (una de cada tres), es decir, personas que se encontraban en situación irregular o con un permiso de residencia de corta duración; sin embargo, al año siguiente, en 2008, los motivos económicos pasan a ocupar la primera posición, al menos en lo que se refiere a los tres primeros trimestres (representan el $46 \%$ de las respuestas negativas). Anteriormente se ha apuntado el coste que supone para muchos inmigrados marroquíes la realización de visitas a su país de origen. Nos estamos refiriendo a meses en los que la crisis económica empezaba a cobrar visibilidad. Posiblemente estas familias aplazaron viajes proyectados en momentos que auguraban mejores expectativas.

Durante la última fase del trabajo de campo y en algunas visitas esporádicas efectuadas en 2009 tuvimos oportunidad de conocer otras realidades. Algunos inmigrados optaron por prolongar sus estancias en Marruecos -o en todo caso la del cónyuge y los hijos- ante las contrariedades sobrevenidas por la pérdida de empleo. Permanecen en los lugares de origen a la espera de que el contexto mejore, y en el caso de los trabajadores agrícolas a que empiece la nueva temporada. De manera general, esto ha tenido lugar durante los meses de verano o aprovechando algunas fiestas de carácter religioso. Como señala Cohen (2009: 12), «estas prácticas son más factibles cuanto más se refuerza el estatus administrativo del inmigrante en España, y que cuanto favorezca su fragilización tendería a bloquearlas». Por ejemplo, es más factible prolongar la estancia en Marruecos para una persona que dispone de un permiso de como mínimo dos años de duración que para otra que únicamente lo tiene de uno, ya que ésta última intenta evitar que la autorización caduque durante su ausencia.

En conclusión, consideramos que en la lectura final de los resultados es conveniente cotejar, por un lado, los valores referentes a los años 2006 y 2007, y por otro, entre éstos y 2008. En la comparación de los dos primeros años apenas se detectan diferencias en las respuestas. Homogeneidad que nos está sugiriendo que los inmigrados adoptan una pauta regular en sus visitas a Marruecos. Este argumento se ratificará a continuación con la secuencia de la distribución a lo largo del año de estos desplazamientos durante el trienio estudiado. Los cambios que se detectan en 2008, en un contexto económico y laboral diferente del que había sido al inicio de la encuesta, dan pie a distintas estrategias para hacer frente a los escollos que afloran: algunos interrumpen las visitas a sus familiares en Marruecos y otros optan por alargarlas provisionalmente.

\section{El calendario de las visitas a Marruecos: diferencias inter-municipales}

Algunos de los entrevistados afirmaron que uno de los criterios que barajaron para cambiar de residencia dentro de España fue el de acercarse a Marruecos y facilitar sus visitas a sus lugares de procedencia. Por ejemplo, es el caso de un joven entrevistado en Albuñol que volvió a la provincia de Granada después de varios años de residencia en Barcelona trabajando en la construcción. Los contrastes territoriales en lo que respecta a la frecuencia de estas visitas se registran también dentro de Andalucía. Ciñéndonos de nuevo a los datos correspondientes al año 2006, Albuñol y, sobre todo, Granada arrojan los promedios más altos de viajes realizados a Marruecos (1,58 y 1,65, respectivamente); Aguilar de la Frontera se sitúa justo en el valor que obtiene el total de encuestados en los cinco municipios estudiados $(1,44)$; en fin, las últimas posiciones corresponden a Zafarraya $(1,26)$ y Lucena $(1,18)$. La secuencia del reparto de las visitas realizadas en los tres años estudiados nos permitirá apreciar con mayor nitidez estos contrastes intermunicipales. 
El procedimiento que hemos adoptado para representar la distribución de los retornos temporales a lo largo del año ha sido el siguiente: se han dividido los desplazamientos correspondientes a cada mes entre el total de personas que viajaron a Marruecos en el año en cuestión. Cabe señalar que muy raramente una persona hizo dos viajes o más en un mismo mes. Esta fórmula ha sido aplicada para los tres periodos a los que se hizo referencia en cada fase de encuesta (2006, 2007 y 2008). Hacemos en primer lugar un inciso en los resultados obtenidos para la suma de encuestados en los cinco municipios. Como podemos observar en la figura 3, en los tres años estudiados las estancias en Marruecos se concentraron mayoritariamente en los meses de verano. Diciembre supuso otro momento clave en los desplazamientos por la coincidencia de la fiesta de Aid El Kebir y las vacaciones de Navidad. También podemos poner de relieve el pequeño pico que aparece en el mes de abril con motivo de la Semana Santa. A la vista de estos datos, podemos afirmar que las prácticas de movilidad de los marroquíes residentes en Andalucía guardan bastante similitud, en lo que al calendario se refiere, de las que llevan a cabo los inmigrados en otros países europeos como Francia, para quienes igualmente los meses estivales representan el momento clave en los retornos temporales. No obstante, la intensidad del trasiego durante los otros meses del año es también semejante.

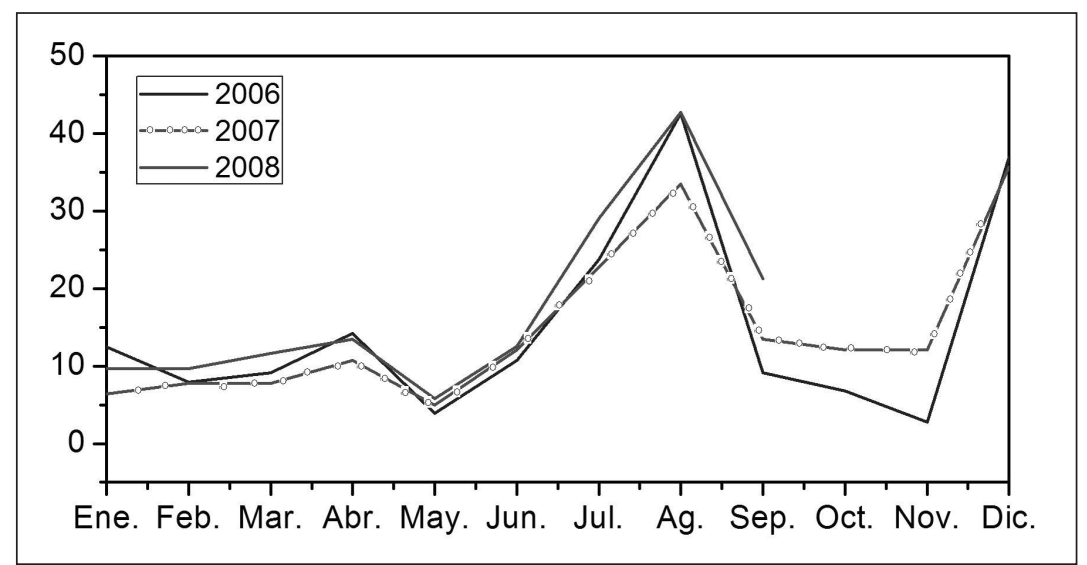

FIGURA 3. Visitas realizadas en 2006-2008 clasificados por meses (\%)

Fuente: Encuesta DINAMO.

Cuando pasamos a observar los datos a escala local en función de los lugares de residencia en Andalucía advertimos que las secuencias temporales que se dibujan difieren entre unos y otros. En los municipios cordobeses las curvas siguen una evolución bastante discontinua, aunque en los dos casos el punto álgido de los movimientos se ubica en el mes de agosto (figura 4). En Lucena los trabajadores en el sector de la industria del mueble se ven abocados a separar las estancias en Marruecos, por lo común, en dos momentos. Difícilmente las empresas conceden más de dos semanas seguidas de vacaciones. La aspiración más extendida, sin embargo, es reunir todos los días disponibles para poder para pasar una temporada más larga en los municipios de origen, a ser posible en periodo estival. Generalmente estos trabajadores optan por unos días en verano y otros en Semana Santa o en Navidades. Nótese que los desplazamientos en Aguilar de la Frontera durante el mes de diciembre son menos frecuentes ya que la presencia de trabajadores en la campaña del 
olivar es más importante que en Lucena. Cabe destacar, por último, el pequeño pico que se repite en 2006 y 2007 en el mes de octubre, y que corresponde a las mismas personas que tradicionalmente pasan el final del Ramadán en Marruecos y que no viajan durante el verano porque se dedican al comercio ambulante por ferias y romerías por varias provincias andaluzas.

Las oscilaciones en la capital granadina son aún más pronunciadas. La circulación entre Marruecos y Andalucía es más frecuente a lo largo del año, aunque también aquí es claro el predominio de unos meses sobre otros: agosto, diciembre y, más modestamente, abril son los que sobresalen especialmente. La mayor diversidad de los perfiles sociolaborales (entre los que tenemos que hacer una mención especial a los comerciantes), la presencia de jóvenes estudiantes que regresan al hogar familiar en los periodos festivos, la proximidad geográfica (Tánger, Tetuán, Nador, Alhucemas... son algunas de las principales áreas de origen)... explican que la frecuencia de los retornos sea mayor entre los marroquíes residentes en la capital granadina que la de los encuestados en los otros municipios.

Esta mayor fluidez en la circulación migratoria entre los dos países en la capital granadina se hace más patente aún si tomamos como punto de partida del movimiento los lugares de origen, es decir, cuando son los familiares en Marruecos los que visitan a los inmigrados en los distintos municipios en los que residen en España. En la primera fase de encuesta se preguntó a los encuestados si algún miembro de la familia los había visitado desde su llegada a España. Granada es el municipio que arroja, con un gran margen de diferencia, el porcentaje más alto de respuestas positivas: así lo reconoció el 63,5\% de la muestra (el 50\% en Lucena y Aguilar de la Frontera, el 37\% en Zafarraya y sólo un 29\% en Albuñol). La cuestión volvió a ser planteada en el último cuestionario, pero en esta ocasión haciendo únicamente alusión a las visitas de los familiares durante el año 2008. De nuevo Granada ostenta, con holgura, el primer puesto en lo que a respuestas positivas se refiere, lo que nos está indicando que, muy posiblemente, son viajes que se repiten cada año. Aquí tienen especial protagonismo los padres de los jóvenes emigrados con un visado de estudios. En algunos casos se está haciendo referencia exclusivamente a una estancia inicial en el primer año de instalación en la ciudad. Pero en otros, efectivamente, son visitas que se reiteran cada año. Es interesante destacar que, en determinados casos, se ha pasado de una circulación muy frecuente entre los dos países, a una instalación semi-permanente en Granada. Esto ocurre sobre todo en familias donde son varios hijos los que se encuentran en Andalucía estudiando o trabajando. Un ejemplo ilustrativo lo encontramos en un joven rifeño, de 34 años, que llegó a Granada en 1992, donde ya se encontraba estudiando un hermano mayor. A posteriori, ya en la década 2000, se incorporan otros hermanos y un poco más tarde también el padre, quien empieza a pasar algunas temporadas en España vinculadas a una actividad comercial. Todos estos movimientos han dado lugar a que en los últimos años la familia se encuentre dividida entre los dos países, con más miembros en España que en el municipio marroquí de origen. Las expectativas ante el futuro en el momento de la entrevista (Granada, mayo de 2008) eran las siguientes: «Hombre, alli no lo vamos a dejar nunca, o sea, nunca vamos a dejar sola en casa a nuestra madre alli y nos vamos a poner a vivir aquí, pero creo que mi madre va a estar aquí más que allí, aquí yo que sé, la mitad del año aquí y la mitad del año allí, como mínimo [...]. Le falta poco para la jubilación [al padre], le falta, a lo mejor, hasta el comienzo del 2009, creo que le falta hasta el comienzo del 2009 o algo así, le falta un poquito, pero mi padre no va a parar nunca, mi padre no creo que pare nunca, mi padre necesita hacer eso, necesita moverse, hacerse sus trapicheos, como ha ido haciendo toda su vida, nunca ha tenido un trabajo al cien por cien, nunca se dedicaba al pescado al cien por cien, siempre ha tenido otros trapicheos paralelos, es decir, 

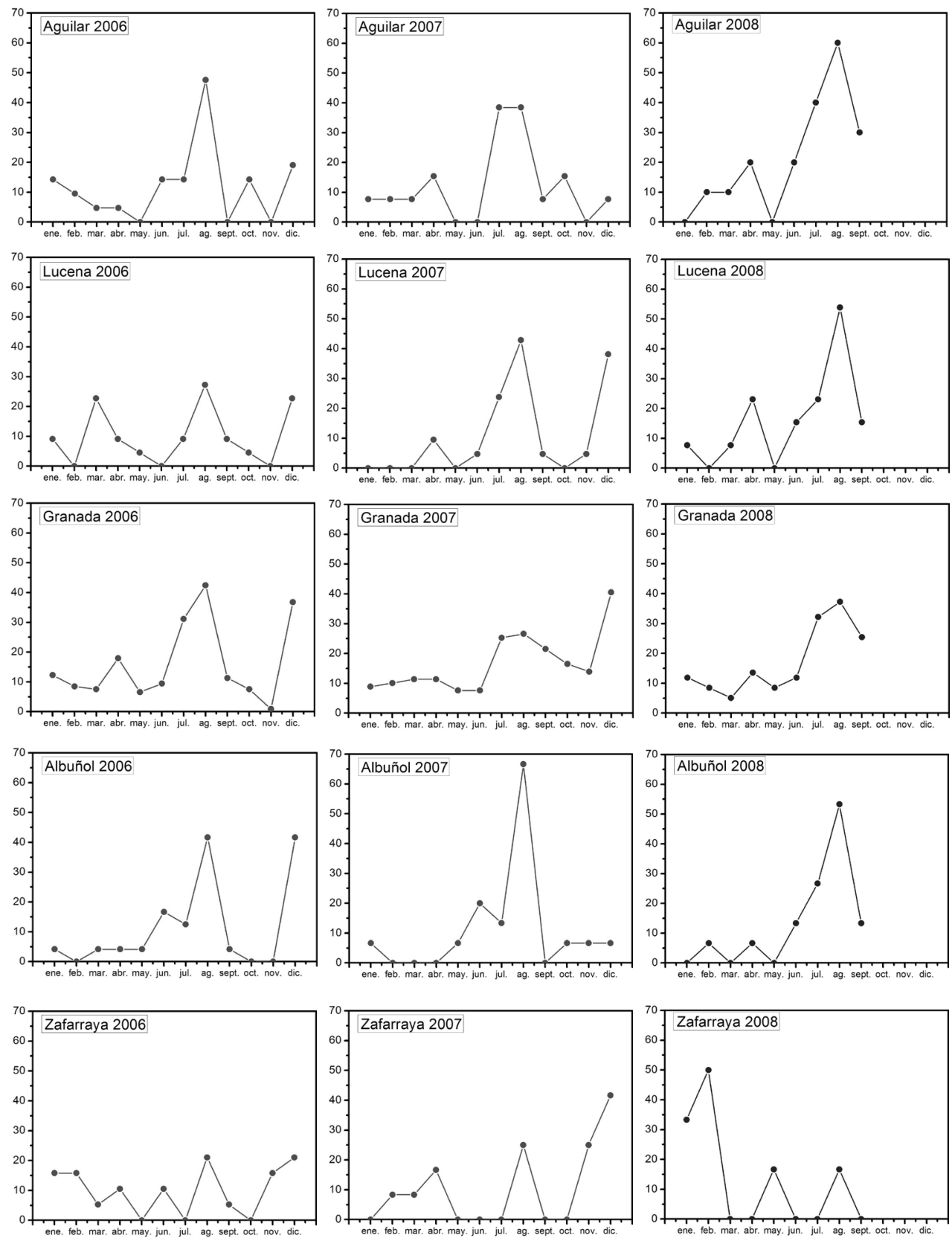

FIGURA 4. Visitas en 2006, 2007 y 2008 para los encuestados en cada municipio (\%)

Fuente: Encuesta DINAMO. 
comprarse un coche y venderlo, todo son negocios para él, todo, comprar redes para barcos, también se dedicaba a eso, para volver a venderlas... [...].Mi madre instalarse no creo que tenga ganas, no creo, ella ha vivido toda su vida alli y allí vive en el centro de Alhucemas, su casa es como la parada obligada de muchas mujeres amigas suyas, ¿no?, que cada día viene una, mi madre es su vida, son sus amigas, sus familiares, las tías, las primas y allí lo tiene todo, aquí no va a ser lo mismo. Aquí nada más que sus hijos, o sea, quiere a sus hijos, pero tampoco puede prescindir de lo otro».

En la secuencia de Albuñol, la aglutinación de las estadías temporales en los meses de verano es aún más evidente. Corresponde al periodo de temporada baja en las tareas agrícolas, tanto para los asalariados como los autónomos. El resto del año se van sucediendo las campañas, con desigual concentración de las jornadas de trabajo según los periodos. Por último, en lo que respecta a Zafarraya (donde la campaña agrícola se desarrolla fundamentalmente en verano), si bien las líneas siguen un despliegue intermitente a lo largo del año, los picos más altos se concentran en los meses de invierno, ya sea a finales del año en cuestión o inicios del siguiente, es decir, los retornos empiezan a organizarse una vez ha finalizado la temporada, por lo común a finales de octubre o principios de noviembre. Cabe señalar, a este respecto, que las familias en Zafarraya encuentran más dificultades para poder extender los días de vacaciones en Marruecos que si se realizasen en verano, ya que el periodo no lectivo en los centros de enseñanza es mayor.

\section{Conclusión}

Se ha comprobado que hay una serie de factores que inciden en que los desplazamientos anuales a Marruecos se hagan con mayor o menor frecuencia, y en diferentes momentos del año. En primer lugar, la procedencia geográfica: no cabe duda de que los oriundos de las provincias de Tánger, Tetuán o Nador disponen de más ventajas en relación con la distancia, el tiempo y el coste de los viajes que los que proceden del Marruecos central o del cinturón urbano atlántico. En segundo lugar, la situación ocupacional: los trabajadores en el sector agrario son los que menos estancias pasan en Marruecos, lo que se explica, en parte, porque algunas de estas personas se desplazan a otros municipios (más o menos próximos) siguiendo el ciclo agrícola anual; por el contrario, los empleados en la construcción o en la industria mueble sí pueden, generalmente, ajustar las estadías en los municipios de origen al calendario festivo español, lo que no siempre se percibe, sin embargo, como una ventaja o privilegio. En tercer lugar, cabe destacar también que las mayores dificultades para viajar a Marruecos se concentran en las primeras fases migratorias: en la mayor parte de los casos porque los inmigrados no han logrado regularizar su situación administrativa, aunque también puede ocurrir que se esté en proceso de búsqueda de un lugar definitivo donde instalarse, un trabajo que ofrezca cierta estabilidad... Esta etapa de exploración puede llevar a algunos jóvenes incluso a viajar al extranjero para intentar captar más oportunidades.

\section{Bibliografía}

CASTLES, S. (2004): «Globalización e inmigración», en Inmigración y procesos de cambio. Europa y el Mediterráneo en el contexto global. Icaria/Temed. Barcelona, pp. 33-56.

CHAREF, M. (1999): La circulation migratoire marocaine : un pont entre deux rives. Editions Sud Contact. Agadir.

CHAREF, M. (2003): «Des hommes passarelles entre l'Europe et le Maghreb», en Hommes et Migrations, $\mathrm{n}^{\circ} 1242$, pp. 6-18. 
COHEN, A. (2009): «España en la encrucijada migratoria (trans-)mediterránea. Una revisión sociogeográfica», en Cahiers de civilisation espagnole contemporaine, $\mathrm{n}^{\circ} 4$. Disponible en: http://ccec.revues.org/index2718.html.

COHEN, A.; CAPOTE, A. (2011): «Las relaciones con Marruecos según los inmigrados a Andalucía», en De Marruecos a Andalucía: migración y espacio social. Editorial Universidad de Granada, Granada, pp. 258- 281.

DE TAPIA, S. (2002): «Immigrations turques en Europe : typologies des espaces et des réseaux», en Les Dossiers de l'IEFA, n 2002, pp. 30-77.

DOUICICH, M. (2007): «Les liens économiques et sociaux des Marocains résidant à l'étranger avec les pays d'origine et d'accueil», en Les marocains résidant à l'étranger. Enquête de 2005 sur l'insertion socio-économique dans les pays d'accueil. HautCommissariat au Plan, Rabat, pp. 197-271.

ESCRIVÁ, Á.; RIBAS, N. (2004): «La investigación sobre migración, desarrollo y transnacionalismo: contribuciones para un debate desde España», en Migración y desarrollo. CSIC, Madrid, pp. 11-51.

SCHAEFFER, F. (2002): «Ici et la-bas : le champ des possibles», en Quand l'alterité se fait en-jeux. L'Harmattan, París, pp. 213-261.

SIMON, G. (1995): Géodynamique des migrations internationales dans le monde. Presses Universitaires de France, París, 1995.

SUÁREZ, L. (2008): «La perspectiva transnacional en los estudios migratorios. Génesis, derroteros y scurso metodológicos», en La inmigración en la sociedad española: una radiografía multidisciplinar. Bellaterra, Barcelona, pp. 771-796.

TARRIUS, A. (20029: La mondialisation par le bas. Les nouveaux nomades des économies souterraines. Balland, París, 2002. 